\title{
Smart Online Transactions Using Voice Recognition for EIderly People
}

\author{
Tanveer Ahmed ${ }^{1}$, Abdul Saleem Javeed ${ }^{2}$, Gunti Spandan ${ }^{3}$, Dr. C. Gopala Krishnan ${ }^{4}$, Beena G Pillai ${ }^{5}$ \\ ${ }^{1}$ Assistant Professor, Dept. of CSE, GITAM (Deemed to be University), Bengaluru, India, tahmed@ gitam.edu \\ ${ }^{2}$ Assistant Professor, Dept. of CSE, GITAM (Deemed to be University), Bengaluru, India, ajaveed@ gitam.edu \\ ${ }^{3}$ Assistant Professor, Dept. of CSE, GITAM (Deemed to be University), Bengaluru, India, sgunti@gitam.edu \\ ${ }^{4}$ Associate Professor, Dept. of CSE, GITAM (Deemed to be University), Bengaluru, India, gchandra@ gitam.edu \\ ${ }^{5}$ Assistant Professor, Dept. of CSE, GITAM (Deemed to be University), Bengaluru, India, bpillai@ gitam.edu
}

\begin{abstract}
The work in this paper targets creating voice based financial framework. In the realm of present day innovation where we use web and advanced mobile phones to modify the effect by building up an function that may really help the individuals who need it the most. We are discussing the provincial pieces of our nation, which makes the greater part of the India. The significant motivation behind why a large portion of these individuals are not yet adjust with this cutting edge innovation is on the grounds that they think that its hard to utilize and don't have a thought of utilizing these advanced cells. This application causes them access the fundamental financial administrations in the manner they are most agreeable.

That is letting them utilize the telephones with voice order control, accessible in their own local language. Particularly the approach of versatile banking, it tackled the issue of going from their town to close by city simply realize the bank balance. This spares their time, additionally telling them about the better offers accessible just on one order.
\end{abstract}

Key words: Voice-based, Banking system, Smart phone, Mobile.

\section{INTRODUCTION}

In a period where we are in a situation of the web and advanced cells, we are attempting to change the effect by building up an application that may really help the individuals who live in rustic pieces of our nation, which makes the greater part of the Indian populace particularly the senior residents. As indicated by the factual report of populace statistics over $70 \%$ of the senior individuals live in country territories that is, there are in excess of 104 million old individuals in India and in excess of 250 million individuals are outwardly tested in India. For the improvement of the general public by making the new innovation simpler to utilize particularly for senior individuals or the truly tested individuals, we have concocted the possibility that voice-based application where client can think about their parity sum or the annuity with voice rather than manual composing. The significant motivation behind why a large portion of these individuals are not yet received with these cutting edge advancements is on the grounds that they think that its hard to utilize and don't have a thought of how these advances functions.

In this paper presents change from text to discourse just as discourse to message for the old matured individuals. By utilizing this operation client can enquire about their bank balance and different administrations. It utilizes text to discourse change and discourse to message transformation where discourse is taken as contribution to arrange whether the client needs the data with respect to adjust or the annuity, at that point from the gained information the equalization or the benefits from the necessary record number is sent to the client in type of discourse. This application additionally basically helps the senior the vast majority who can't see and the individuals who doesn't see some other language other than their native language.

In our proposed framework client can get to their bank parity and annuity through voice where the client requests their equalization and benefits for which the framework answers by soliciting the record number from the client. After getting the record number the framework rehashes the record number for approval and inquires as to whether the record number gave by the client is right or mistaken, if the client says the record number is erroneous again the record number is inquired as to whether the number 
is right it is coordinated with the record number put away in the information base, if the match is effective the mentioned balance is gotten by the client in type of discourse.

In our proposed structure input is given in neighborhood language which is changed over into close by language text through Google Translate API which gives English substance as response which is seen by the machine about the request presented by the customer whether it is equality or annuity, by then machine represents the request as for the record number and the record number is replied in close by language which is changed over into neighborhood language text which is again changed over into English substance and is shipped off the specialist. The response obtained from the laborer is in English substance determining if the customer needs equality or advantages then the English substance is gotten from the specialist is shipped off Google Translate API which is then changed over into English talk.

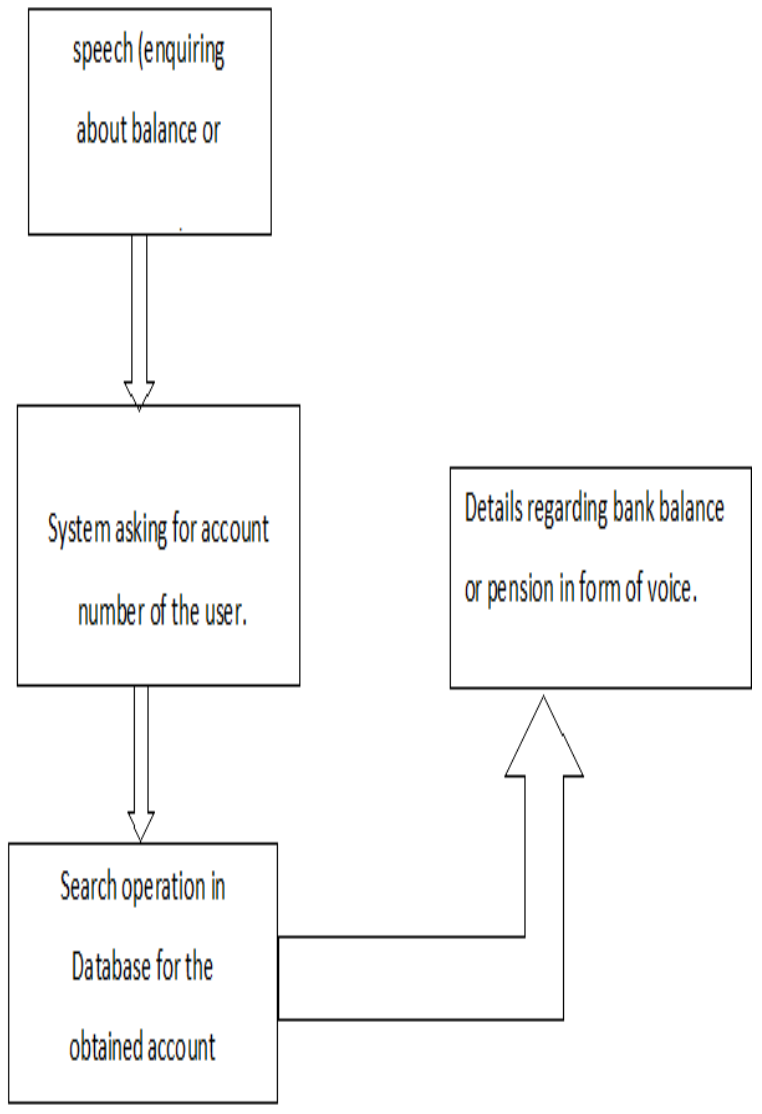

Figure1:- Summary of proposed system
Figure1 shows the system which is arranged in this endeavor. A first bank equality or annuity detail is requested in structure from talk. Text to talk (TTS) and talk to text is used then from the data base record number is composed with the record number gave by the customer achieving the bank nuances in kind of talk.

\section{LITERATURE SURVEY:-}

\subsection{Text to speech based on FFT:-}

This framework presents French content to discourse framework which depends on diaphone connection measure in which text to discourse combination calculation is utilized to separate the sentence into words and again into letter sets which is then linked into a sentence shaping a discourse [4].

\subsection{Text to phoneme conversion:-}

This paper utilizes the repetitive neural organizations prepared with the ongoing intermittent learning (RTRL) calculation which endeavors to perform text-to-phoneme change by utilizing intermittent neural organizations prepared with the continuous repetitive learning (RTRL) calculation [6].

\subsection{Speech to text conversion using CMU sphinx:-}

This framework presents the issue of discourse to message change of Kannada Language. In this framework they have propose a Kannada Automated Speech to Text change System. They have prepared and test the Speech Processing System utilizing CMU Sphinx structure [7].

\subsection{Speech to text and speech to speech summarization of spontaneous speech:-}

This framework presents discourse to-text and discourse to-discourse programmed rundown dependent on discourse unit extraction and link where sentences are destitute in words and afterward to single characters in digitalized structure [8].

\subsection{Text conversion for multilingual languages:-}

This paper presents a multilingual discourse to-message transformation framework. Discourse is the regular and most significant type of correspondence for person. Discourse To-Text (STT) framework accepts a human discourse expression as information and requires a series of words as yield [9].

\subsection{Text to speech synthesis system in Indian}




\section{English:-}

In this framework they altered the current English grapheme to phoneme word reference by actualizing specific governs for one specific assortment of Indian English, specifically Assamese English. The proposed technique for word reference modification is applied at the front finish of the Indian English TTS[14].

\section{SYSTEM DESIGN}

\section{The main function of the paper is shown in this}

\section{diagram.}

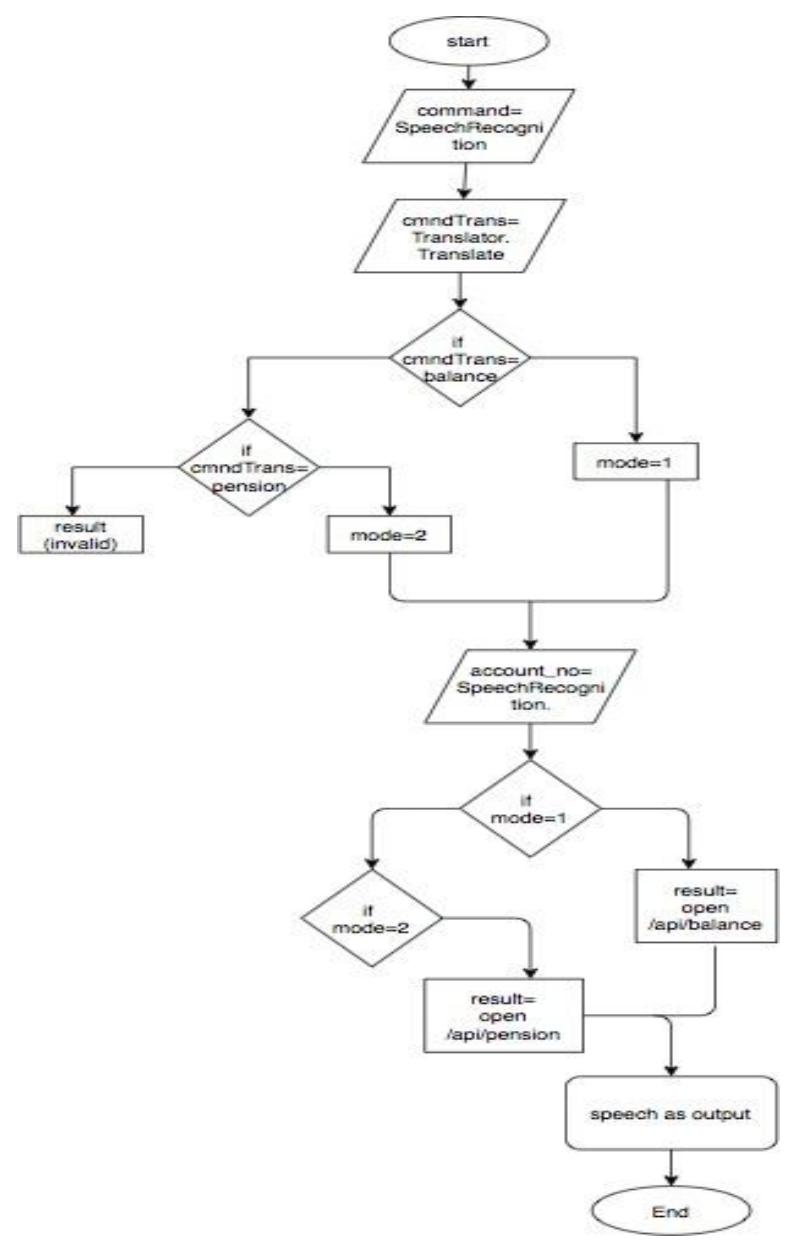

Figure 2:- Main Function

As individual deals the application for assistance through voice. Application sees the discussion from the individual and stores it in a record. The report ought to have two or three plans like it ought to be a WAV record encoded into LINEAR16 structure correspondingly called as LINEAR PCM. Right when application calls outer converse with message API, the application begins analyzing the reaction byte by byte and stores it in a variable. Considering the mentioning from the client either for balance or for annuity application calls express worker end point. As reaction from API is changed over into talk. The solicitation given by the client is seen and changed over into English language which is seen by the machine subordinate upon the system for activity the outcome can be balance aggregate, benefits or the invalid record number. Machine sees the discussion occurring into the client mentioning of getting to the parity sum or the points of interest which is returned in sort of talk.

\section{IMPLEMENTATION}

Here undertaking utilizes content to discourse transformation (TTS) along with discourse via message change where discourse in taking information and discourse is perceived distinguishing whether the client needs the data with respect to adjust or the annuity, at that point from obtained input the equalization or the benefits from necessary record digit is transmitted to the client in type of discourse i.e. in this task, client approaches the framework for balance subtleties or the benefits in type of discourse, consequently the framework asks the record digit. This record digit is looked in the information base restoring the subtleties of bank parity or annuity in type of text which will later be changed over into the discourse that can be effectively perceived by client coming up next is the square outline showing the usage chunk of the program.

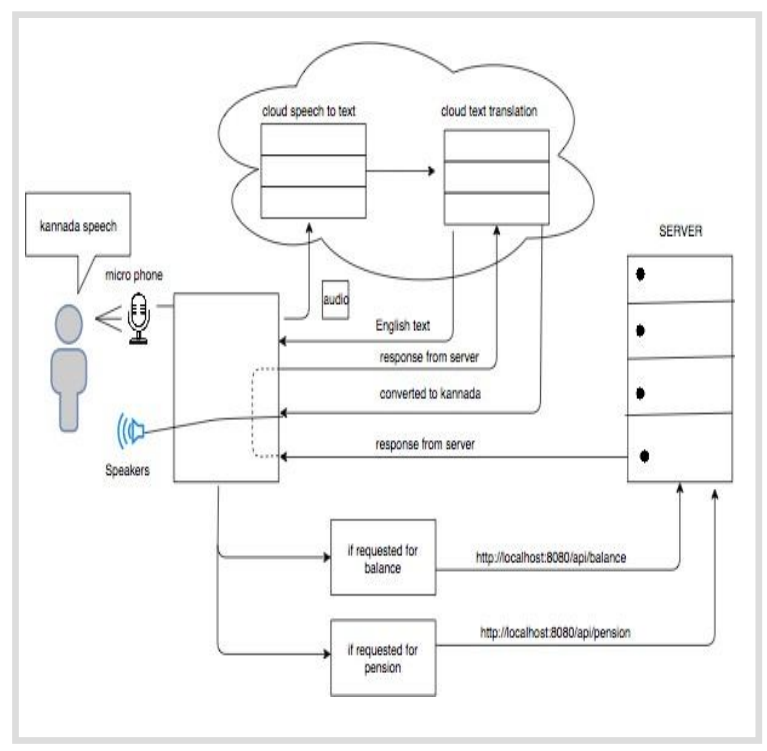

Figure 3:- Description of proposed system

The Cloud Platform API can progressively interpret content bounded by a large numbers of 
conversation sets. An association is formed by making a orderly asset finder from the worker. The discourse recorder is utilized to make the sound documents by recording the substance that the client talks about bank subtleties. The discourse recognizer perceives the sound document. The interpreter of the API makes an interpretation of the discourse to message.

\section{RESULTS}
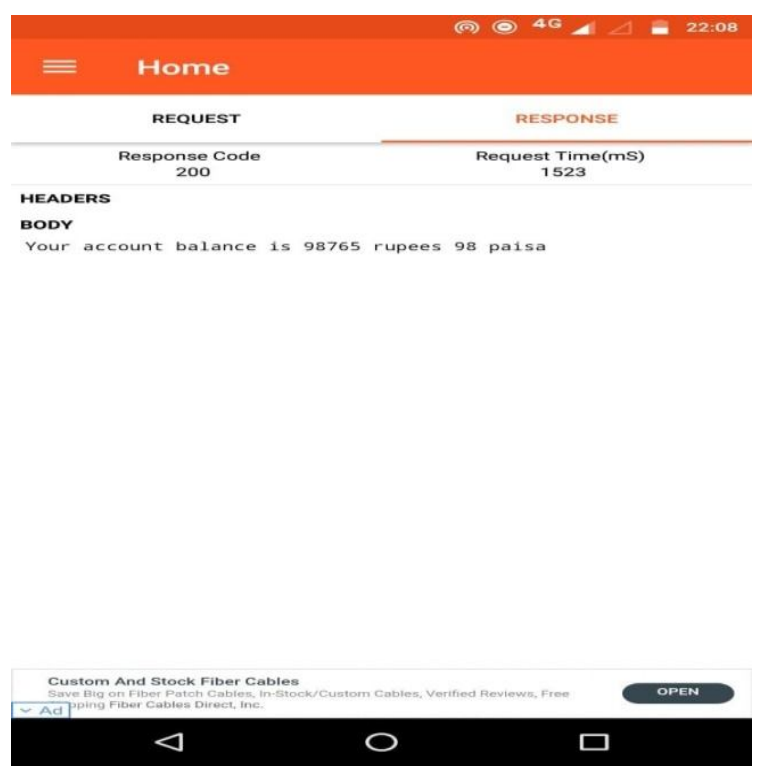

Figure 4:- Getting to adjust subtleties through advanced mobile phone.

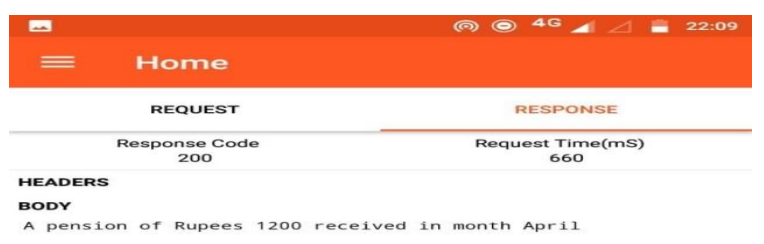

Data Science Fellowship

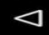
口

Figure 5:- Getting pension details through advanced mobile phone.

\section{CONCLUSION}

In this function we have actualized a few highlights pertinent to improvement of investment frameworks. The content to discourse also discourse to content is productive and is simpler to use for the elderly individuals for inquisitive about their bank balance or required benefits for the elderly folks individuals as well as be valuable for dazzle individuals to think about their record subtleties without going to the bank. An information base is made with account numbers having equalization or annuity where client requests their parity and benefits giving their record number which is then approved adjusting the client bringing about the parity sum and benefits which their record number holds.

\section{REFERENCES}

1. Sanjib Das, "Speech Recognition Technique: A Review", International Journal of Engineering Research and Applications (IJERA) ISSN: 22489622 Vol. 2, Issue 3, May-Jun 2012.

2. Ms. Sneha K. Upadhyay, Mr. Vijay N. Chavda,'Intelligent system based on speech recognition with capability of self learning" ,International Journal For Technological Research In Engineering ISSN (Online): 2347 - 4718 Volume 1, Issue 9, May-2014.

3. Deepa V.Jose, Alfateh Mustafa, Sharan R,"A Novel Model for Speech to Text Conversion "International Refereed Journal of Engineering and Science (IRJES)ISSN (Online) 2319-183X, Volume 3, Issue 1 (January 2014).

4. Moulines, E,'Text-to-speech algorithms based on FFT synthesis." 1988.

5. Sultana, S.,Akhand, M. A H, Das, P.K., Hafizur Rahman, M.M."Speech-to-Text (STT) conversion using SAPI for Bangla language"2012.

6. Yee-Ling Lu, Man-Wai and Wan-Chi Siu "Text to phoneme conversion" 1995.

7. Shivakumar K.M, Aravind K.G, Anoop T.V,DeepaGupta"Kannada Speech to Text Conversion using CMU Sphinx”2003.

8.SadaokiFurui,Tomonori Kikuchi, YousukeShinnaka, and Chiori Hori, "Speech-to-Text and Speech-to-Speech Summarization of Spontaneous Speech"2004.

9. Yogita H. Ghadage, Sushama D. Shelke "Speech to Text Conversion for Multilingual Languages" 2016.

10. Stephanie Challita, FaiezZalila, Christophe Gourdin, and Philippe Merle Inria Lille "A Precise Model for Google Cloud Platform”2018.

11. Jianliang Meng, JunweiZhang,Haoquan Zhao "Overview of the Speech Recognition Technology" 2012. 
12. Tudor Barbu"Comparing Various Voice

Recognition Techniques" Iasi branch Iasi, ROMANIA,2010.

13. Anurag Mishra, Pooja Makula, Akshay Kumar, Krit Karan and V. K. Mittal "A Voice-Controlled Personal Assistant Robot"2015.

14. Deepshikha Mahanta, Bidisha Sharma, PriyankooSarmah, S R Mahadeva Prasanna" Text to Speech Synthesis System in Indian English",2011. 15. Text-to-speech conversion system using a format based synthesis technique for Brazilian Portijguese, Signal Processing Laboratory of FEEC-Uni camp, 2010 